\title{
Subsistema coparental: revisão sistemática de estudos empíricos
}

\author{
Crístofer Batista da Costa \\ Mariana Rodrigues Machado \\ Mariana Cunha Schneider \\ Clarisse Pereira Mosmann \\ Universidade do Vale do Rio dos Sinos, RS, Brasil
}

\begin{abstract}
Resumo
Estudos sobre coparentalidade são recentes no Brasil, embora a literatura científica investigue o construto há mais de quatro décadas e confirme sua relevância à pesquisa e à clínica psicológica. Neste artigo, objetivou-se analisar as características metodológicas, os objetivos e os resultados de estudos empíricos sobre coparentalidade. Trata-se de uma revisão sistemática de artigos científicos no período 2007-2016, no Portal BVS e nas bases PsycINFO e Web of Science, por meio dos termos coparentalidade e aliança parental. Com base nos critérios de inclusão, 29 artigos foram selecionados para análise. Verifica-se que as pesquisas investigam a coparentalidade por meio de pesquisa quantitativa, longitudinal e explicativa. Apontam a multiplicidade de perspectivas de investigação da coparentalidade que pode estar associada aos questionamentos que ainda buscam ser respondidos e refletir as diferentes formas de compreender os processos familiares e as repercussões no indivíduo e no casal. São discutidos resultados relevantes aos profissionais da clínica e aos pesquisadores.
\end{abstract}

Palavras-chave: Relações familiares; Relações pais-filho; Revisão.

\section{Coparenting subsystem: systematic review of empirical studies}

\begin{abstract}
Studies about coparenting are recent in Brazil, although the scientific literature has investigated the construct for over four decades and confirmed their relevance for research and clinical psychology. The objective of this study was to analyze the methodological characteristics, the objectives, and the results of empirical studies about coparenting. This is a systematic literature review, from 2007 to 2016, through BVS, PsycINFO, and Web of Science, using the terms "coparenting" and "parental alliance", in Portuguese and English. Based on inclusion criteria, 29 articles were selected for analysis. The studies investigated coparenting with quantitative longitudinal and explanatory designs. They point to the multiplicity investigative perspectives on coparenting, which may be associated to the questions that are yet to be answered and reflect the different ways to understand family processes, as well as the impact on the individual and the couple. Relevant results are discussed for clinicians and researchers.
\end{abstract}

Keywords: Family relationships; Parent-child relationships; Review.

\section{Copaternidad: revisión sistemática de artículos científicos}

\section{Resumen}

Los estudios sobre la copaternidad son recientes en el Brasil, aunque la literatura científica investigar la construcción durante más de cuatro décadas y confirmar su relevancia para la investigación y la psicología clínica. Este estudio tuvo como objetivo analizar las características metodológicas, los objetivos y los resultados de los estudios empíricos sobre la copaternidad. Se trata de una revisión sistemática de artículos científicos en el período 2007-2016 en la BVS, PsycINFO y Web of Science a través de los términos de copaternidad y la alianza de los padres en portugués e Inglés. Con base de los criterios de inclusión, se seleccionaron 29 artículos para el análisis. Se hace notar que los estudios investigan de la copaternidad a través de estúdios cuantitativa, longitudinal y explicativa. Señalan a la multitud de perspectivas de investigación de copaternidad que se pueden asociar a las preguntas que aún tratan de ser contestadas. Y reflejar las diferentes formas de entender los procesos de la familia y el impacto en el individuo y la pareja. Se discuten los resultados relevantes para los professionales de la clinica.

Palabras clave: Relaciones familiares; Relaciones padres-hijos; Revisión. 


\section{Introdução}

Estudos na área de família discutem os reflexos da (dis)funcionalidade da relação conjugal e parental sobre o desenvolvimento dos filhos (Frizzo, Kreutz, Schmidt, Piccinini, \& Bosa, 2005; Grzybowski \& Wagner 2010; Lamela, Figueiredo, \& Bastos, 2010; Lamela, Figueiredo, \& Bastos, 2013; Lamela, Nunes-Costa, \& Figueiredo, 2010), buscando ampliar e clarificar de que forma as interrelações que ocorrem no sistema familiar impactam não só a prole, mas todos os seus membros. Nesse intento, os pesquisadores incluíram ao binômio parentalidade e conjugalidade o subsistema coparental (Feinberg, 2003).

A coparentalidade tem sido postulada como o subsistema mediador entre o relacionamento marital e as relações entre pais e filhos. Essa articulação explicaria o "porquê" e "como" as relações conjugais impactam diretamente na parentalidade e, indiretamente, no comportamento dos filhos (Margolin, Gordis, \& John, 2001).

Embora a coparentalidade seja alvo de pesquisas em outros países há aproximadamente quatro décadas, é uma temática de investigação relativamente recente no contexto brasileiro (Frizzo et al., 2005; Lamela, Nunes-Costa, \& Figueiredo, 2010). Objeto de trabalhos envolvendo temas como divórcio (Grzybowski \& Wagner 2010; Lamela, Figueiredo, \& Bastos, 2010; Lamela et al., 2013), famílias com membros com necessidades especiais (Sifuentes \& Bosa, 2010), e crianças com problemas de ansiedade (Majdandzic, Vente, Sifuentes, \& Bosa, 2012), a coparentalidade tem sido investigada como um elemento explicativo de padrões de interação familiar (Mosmann, Costa, Einsfeld, Silva, \& Koch, no prelo).

Pesquisa empírica sobre o tema indicou que os subsistemas, conjugal, parental e coparental são interdependentes, entretanto, a coparentalidade aparece como fator interveniente entre a parentalidade e a conjugalidade diante de sintomas internalizantes e externalizantes (Mosmann et al., no prelo). Esse resultado demonstra a influência preponderante na saúde mental de crianças e adolescentes (Teubert \& Pinquart, 2010) e o impacto negativo dessa influência por meio do aparecimento de sintomas emocionais e comportamentais na prole.

Identifica-se na literatura diferentes definições de coparentalidade, algumas que se referem ao seu exercício apenas pelo par conjugal, ainda que separados, (Margolin et al., 2001; Teubert \& Pinquart, 2010; Frizzo et al., 2005) e, outras, em que dois adultos, avó e mãe, pai e tia, mão adotiva e tio, não necessariamente um casal, responsabilizam-se pelo cuidado com as crianças e adolescentes que pertencem ao núcleo familiar (McHale, Kuersten-Hogan, \& Rao, 2004; Sifuentes \& Bosa, 2010). Nesse estudo, será utilizada a segunda definição, considerando que se trata de um conceito mais amplo de coparentalidade, e os termos responsáveis ou cuidadores sempre que for feito referência a díade coparental.

A coparentalidade ou aliança parental, termo utilizado em alguns estudos, visa o envolvimento conjunto e a cooperação entre os adultos responsáveis pelas tomadas de decisão e bem-estar dos filhos que supera as tarefas diárias de cuidado. Envolve as atividades que formam o sujeito, estimulam suas habilidades e condutas, o que pode ser desempenhado, corriqueiramente, pela díade, ainda que separadamente. Outras características relevantes são, por exemplo: o apoio e a solidariedade entre os cuidadores, a amplitude da anuência (ou antagonismo) e a harmonia (ou dissonância) no comportamento dos adultos na medida em que ambos participam ativamente do cuidado à criança. Nesse sentido, a coparentalidade materializase quando, ao menos dois indivíduos, na comunhão de esforços e acordo mútuo, responsabilizam-se por uma criança (McHale et al., 2004; Sifuentes \& Bosa, 2010).

$\mathrm{Na}$ relação de coparentalidade não se objetiva aferir sobre aspectos legais, emocionais, sexuais ou financeiros, inerentes à relação dos adultos e que não possuem vinculação direta aos cuidados da prole (Feinberg, 2003). Trata-se, portanto, de um construto relevante para a avaliação e compreensão do comportamento individual dos filhos e da unidade familiar (McDaniel \& Teti, 2012).

Foram encontrados na literatura quatro modelos conceituais sobre coparentalidade (Feinberg, 2003; Margolin et al., 2001; McHale et al., 2004; Van Egeren \& Hawkins, 2004). O Modelo Ecológico da Coparentalidade de Feinberg (2003) propõe integrar os quatro elementos essenciais da coparentalidade no contexto ecológico, são eles: (a) acordo quanto às práticas parentais, (b) divisão de atribuições ou trabalho no trato com a prole, (c) apoio mútuo quanto às contribuições, decisões e autoridade do cuidador frente aos filhos e (d) gestão conjunta da unidade familiar. No modelo de Margolin et al. (2001) a coparentalidade é considerada uma das experiências mais complexas que adultos podem compartilhar, tendo em vista a necessidade de negociação do papel de cuidadores, tais como: apoio, responsabilidades e contribuições que cada um faz em relação aos filhos. Esse modelo envolve as dimensões: (a) nível de conflito, (b) cooperação e (c) triangulação.

No Modelo apresentado por McHale et al. (2004) a coparentalidade constitui-se na comunhão de 
esforços e na cooperação entre adultos que assumem uma função organizadora em prol do cuidado e da educação dos filhos. Os autores articulam quatro elementos que compõe o constructo: (a) divisão de tarefas relacionadas ao cuidado com as crianças, (b) influência da solidariedade e apoio entre a dupla coparental, (c) (des)harmonia e (in)compatibilidade entre os pais acerca das questões que envolvam os filhos como, educação, por exemplo, e (d) alcance e impacto da qualidade do envolvimento entre a díade e suas condutas diretas com a criança (Majdandzic et al., 2012). No último modelo (Van Egeren \& Hawkins, 2004), a coparentalidade ocorre se, ao menos, dois indivíduos, por mútuo consentimento, assumem responsabilidade conjunta pelo bem-estar de uma criança formando uma díade coparental. Os fatores que compõem o modelo são: (a) solidariedade coparental, (b) apoio às qualidades parentais do cuidador, (c) neutralização da parentalidade por meio de ações que maculam a imagem ou autoridade do outro adulto e (d) parentalidade partilhada.

Os quatro modelos conceituais da coparentalidade encontrados na literatura constituem uma base para as pesquisas que estão sendo realizadas. Por outro lado, percebe-se que a investigação do subsistema coparental é recente, a produção científica escassa no contexto brasileiro e as evidências de validade do construto ainda incipientes devido à carência de instrumentos para mensurá-lo e necessidade de aperfeiçoamento daqueles que estão sendo utilizados (Frizzo et al., 2005).

Nesse sentido, dois estudos teóricos sobre o tema da coparentalidade (Lamela, Nunes-Costa, \& Figueiredo, 2010; Frizzo et al., 2005) apresentaram e discutiram as diferentes perspectivas de entendimento conceitual do subsistema coparental e a importância do referido construto para a pesquisa e para a clínica psicológica. Além disso, evidenciaram a necessidade de estudos empíricos sobre a coparentalidade e que testassem as diferentes propostas. Com base no exposto, esta revisão sistemática da literatura tem por objetivo analisar as características metodológicas, os objetivos e os resultados dos estudos empíricos sobre coparentalidade.

\section{Método}

Delineamento: o presente estudo é uma revisão sistemática de artigos científicos de natureza descritiva e crítica. A revisão sistemática é uma estratégia por meio da qual são selecionados artigos com base em critérios de inclusão e exclusão precisos, feita a avaliação crítica e reflexiva dos estudos e a síntese do material, não sendo, portanto, uma apresentação meramente cronológica e/ ou descritiva de estudos (Costa, Zoltowski, Koller, \& Teixeira, 2015; Fernández-Ríos \& Buela-Casal, 2009).

Procedimentos de busca: a pesquisa foi realizada em sete bases de dados: Lilacs, Ibecs, Medline, Biblioteca Cochrane e Scielo (via Portal da Biblioteca Virtual em Saúde - BVS), PsycINFO e Web of Science. Os termos utilizados deveriam constar no título do artigo, sendo eles coparentalidade e aliança parental e seus equivalentes em inglês coparenting e parental aliance, considerando que a busca neste idioma resgatou os artigos publicados em espanhol. Ressalta-se que devido a inexistência de descritores específicos sobre a temática na Biblioteca Virtual em Saúde (Decs/BVS) foram escolhidos como termos de busca nas bases de dados aqueles mais utilizados na literatura científica, segundo a percepção dos autores deste estudo.

Os procedimentos de busca, seleção e análise dos artigos foram realizados por dois juízes independentes em cinco etapas, de acordo com os critérios utilizados na escala para avaliação da qualidade metodológica das revisões sistemáticas (Costa et al., 2015). Etapa (1): busca, nas bases de dados, de artigos científicos das ciências humanas e da saúde, nos idiomas português, espanhol e inglês, no período de 2007 a agosto de 2016 - dez anos, disponíveis em texto completo e que versassem sobre coparentalidade ou aliança parental; Etapa (2): exclusão dos estudos repetidos nas bases de dados e avaliação dos artigos por meio do título, no qual deveria constar ao menos um dos termos utilizados na busca; Etapa (3): análise dos resumos a fim de identificar estudos empíricos que indicassem claramente no objetivo investigar questões de coparentalidade ou aliança parental; Etapa (4): procedimento de concordância entre dois juízes independentes quanto à exclusão e inclusão dos estudos, encaminhamento dos casos discordantes para avaliação do terceiro juiz e realização de consenso; Etapa (5): elegibilidade, descrição, análise e discussão dos estudos pelos juízes.

Análise dos dados: procedeu-se, inicialmente, com uma análise das principais características dos estudos revisados, que foram: ano de publicação, local de realização do estudo, tipo de pesquisa e amostra. Foi realizada também uma análise crítica dos objetivos e dos resultados encontrados nessas pesquisas numa perspectiva horizontal e vertical, verificando a presença de resultados comuns e específicos, respectivamente.

\section{Resultados}

Ao realizar a busca nas bases de dados por meio dos termos coparentalidade e aliança parental em 


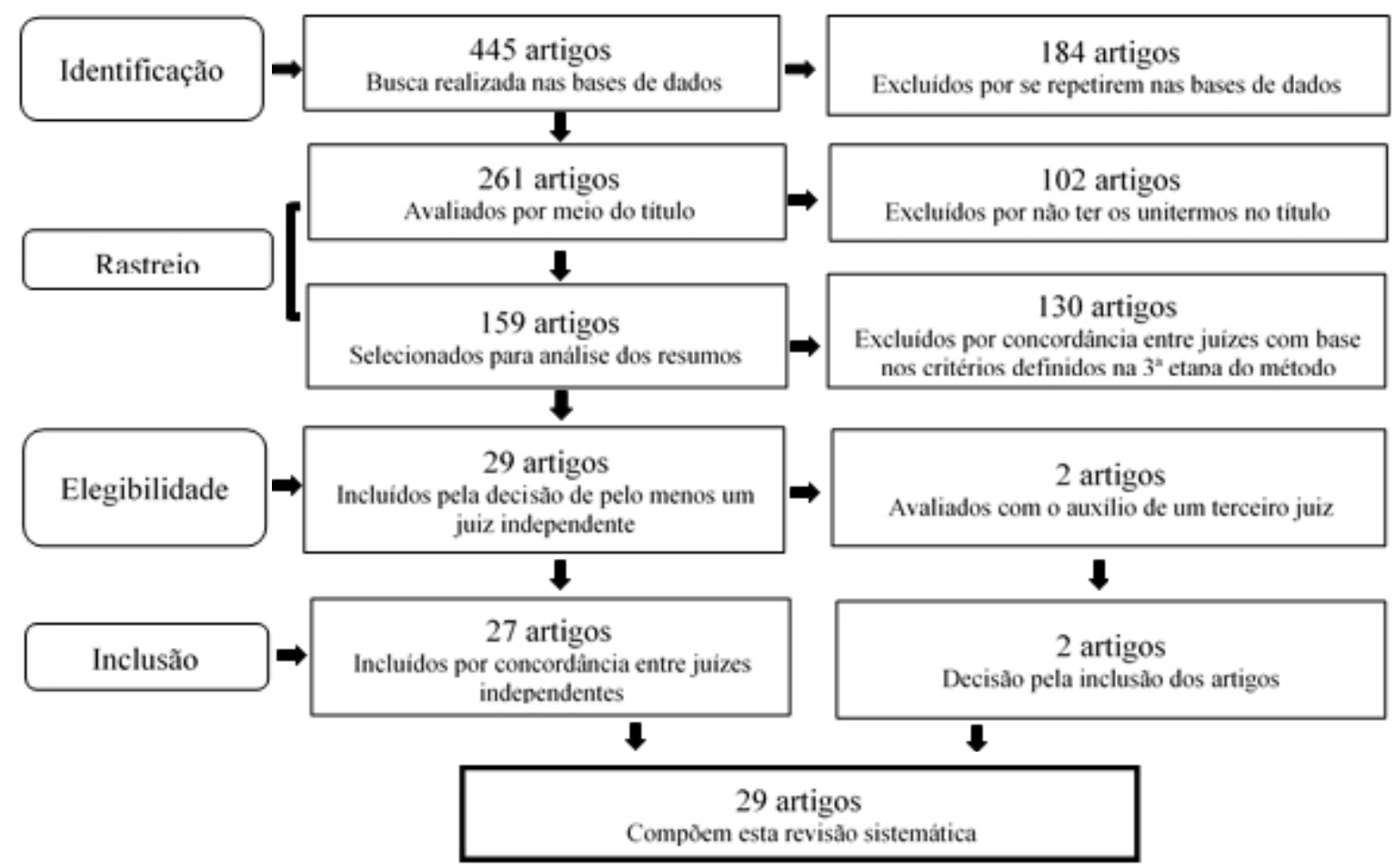

Figura 1. Fluxograma

português e inglês, foram encontrados 445 artigos, conforme o fluxograma acima (Figura 1). A distribuição dos estudos por base ficou da seguinte forma: no portal da Biblioteca Virtual em Saúde - BVS, em que estão as bases Lilacs, Ibecs, Medline, Biblioteca Cochrane e Scielo foram encontrados 153 artigos, na PsycINFO, 52 artigos e na Web of Science, 240 artigos.

$\mathrm{Na}$ segunda etapa do processo foram excluídos 184 artigos que se repetiam entre as bases de dados e mais 102 artigos que, apesar de capturados na busca, não apresentavam os termos coparentalidade ou aliança parental no título. Após essas exclusões, 159 estudos foram selecionados para análise por meio do resumo. De acordo com os critérios definidos na terceira etapa do método, ou seja, estudos empíricos indicando claramente no objetivo investigar questões de coparentalidade ou aliança parental, foram excluídos 130 artigos. As exclusões envolveram os seguintes motivos: estudos teóricos, documentais, revisões de literatura e metaanálise, artigos não disponíveis em texto completo, pesquisas de intervenção, programas de treinamento para pais e estudos para validação de instrumento. $\mathrm{Na}$ etapa final, 27 estudos foram incluídos sob concordância dos dois juízes independentes e dois, sob os quais houve discordância, foram avaliados com o auxílio de um terceiro juiz, definindo-se pela inclusão, e totalizando 29 artigos para análise nesta revisão sistemática.

Na Tabela 1 é possível verificar que entre os anos de 2007 e 2015 foi publicado ao menos um artigo científico por ano sobre o exercício da coparentalidade, sendo que neste período o maior número de publicações se concentrou em 2007 e 2013, em que foram registrados cinco artigos em cada ano. Em 2016 até o mês de agosto, em que se realizou a busca, não há registro de artigos nas bases utilizadas. Com relação ao local de realização dos estudos, os Estados Unidos destacam-se com relação aos demais países tendo realizado 25 pesquisas, seguido da Holanda com duas, do Brasil e de Portugal, ambos com uma pesquisa. Com relação ao idioma dos estudos, foram encontramos apenas artigos publicados em português e inglês.

Nas características metodológicas, descritas na Tabela 2, chama atenção a diferença entre o número de pesquisas quantitativas, 26 estudos, em relação às qualitativas, três estudos. Entre elas, nove foram pesquisas transversais e vinte foram pesquisas longitudinais. Em termos de delineamento, dezenove estudos foram explicativos, seguido de um estudo explicativo e também comparativo, dois estudos correlacionais e três estudos descritivos. No que refere aos participantes, os estudos investigaram casais heterossexuais com as seguintes características: casais gestantes (quatro), casais com filho recém-nascido (cinco), casais com filho pequeno (doze), casais com filho adolescente (quatro), casais com dois filhos, mas sem especificação de idade (dois), pais divorciados (dois).

O objetivo principal dos estudos, conforme Tabela 3, apresentam diversificadas perspectivas 
de investigação. Evidencia-se o interesse dos pesquisadores em analisar o impacto do temperamento ou do comportamento infantil sobre a coparentalidade e a conjugalidade, alterando o exercício coparental e parental e a percepção dos pais e os níveis de satisfação conjugal (estudos identificados pelo Id: $1 ; 3 ; 5$, 7; $10 ; 11 ; 13 ; 15 ; 19 ; 20 ; 23 ; 26 ; 28)$. Outros estudos associaram problemas no comportamento infantil com estresse familiar, depressão, angústia nos pais e a coparentalidade $(6 ; 14 ; 17)$.

Outra onda de pesquisas avaliou os subsistemas conjugal, parental e coparental sem incluir a prole (4; $9 ; 16 ; 18 ; 21 ; 22 ; 27 ; 29)$ focalizando questões como conflitos conjugais e resolução de problemas, satisfação conjugal, responsabilidade, apoio e confiança coparental, eficácia parental e sentimentos em relação aos filhos. A perspectiva de avaliação da relação entre o casal antes e depois da chegada do filho foi investigada por meio das expectativas dos pais sobre a relação familiar e a estabilidade do apoio coparental $(2 ; 24)$, comparando a coparentalidade no primeiro e no segundo filho (15), e quanto às crenças das mães sobre o papel coparental dos pais (22). As diferenças entre o pai e a mãe no exercício da coparentalidade foi objetivo em três estudos $(3 ; 8$; 14) e, por fim, dois estudos $(12 ; 25)$ tiveram como foco a coparentalidade no contexto do divórcio.

Os resultados dos estudos que constam na Tabela 3 foram divididos em quatro perspectivas: (a) qualidade da relação conjugal como preditora da qualidade da relação coparental, (b) variáveis que contribuem

TABELA 1

Caracterização dos estudos

\begin{tabular}{|c|c|c|c|}
\hline Id. & Título & Ano & País \\
\hline 1 & Goodness-of-fit in family context: Infant temperament, marital quality, and early coparenting behavior & 2007 & Estados Unidos \\
\hline 2 & Is seeing believing? Expectant parents' outlooks on coparenting and later coparenting solidarity & 2007 & Estados Unidos \\
\hline 3 & Processes linking adolescent well-being, marital love, and coparenting & 2007 & Estados Unidos \\
\hline 4 & Parental expressiveness as a moderator of coparenting and marital relationship quality & 2007 & Estados Unidos \\
\hline 5 & The longitudinal influence of coparenting conflict on parental negativity and adolescent maladjustment & 2007 & Estados Unidos \\
\hline 6 & Family-level coparenting processes and child gender as moderators of family stress and toddler adjustment & 2008 & Estados Unidos \\
\hline 7 & Parenting, coparenting, and effortful control in preschoolers & 2008 & Holanda \\
\hline 8 & Maternal gatekeeping, coparenting quality, and fathering behavior in families with infants & 2008 & Estados Unidos \\
\hline 9 & Withdrawal from coparenting interactions during early infancy & 2008 & Estados Unidos \\
\hline 10 & Are some children harder to coparent than others? Children's negative emotionality and coparenting relationship quality & 2009 & Estados Unidos \\
\hline 11 & The role of infant temperament in stability and change in coparenting across the first year of life & 2009 & Estados Unidos \\
\hline 12 & Casa do pai, casa da mãe: A coparentalidade após o divórcio & 2010 & Brasil \\
\hline 13 & Links between mothers' and fathers' perceptions of infant temperament and coparenting & 2010 & Estados Unidos \\
\hline 14 & Mother and father adjustment during early parenthood: The roles of infant temperament and coparenting relationship quality & 2011 & Estados Unidos \\
\hline 15 & And baby makes four: The stability of coparenting and the effects of child temperament after the arrival of a second child & 2012 & Holanda \\
\hline 16 & $\begin{array}{l}\text { Associations among marital qualities, supportive and undermining coparenting, and parenting self-efficacy: Testing } \\
\text { spillover and stress-buffering processes }\end{array}$ & 2012 & Estados Unidos \\
\hline 17 & Coparenting quality during the first three months after birth: The role of infant sleep quality & 2012 & Estados Unidos \\
\hline 18 & Marital satisfaction and partners' parenting practices: The mediating role of coparenting behavior & 2012 & Portugal \\
\hline 19 & Cooperative coparenting moderates the association between parenting practices and children prosocial behavior & 2013 & Estados Unidos \\
\hline 20 & $\begin{array}{l}\text { Links among coparenting quality, parental gentle guidance, and toddlers' social emotional competencies: Testing direct, } \\
\text { mediational, and moderation models }\end{array}$ & 2013 & Estados Unidos \\
\hline 21 & Maternal and paternal perceptions of coparenting as a link between marital quality and the parent-toddler relationship & 2013 & Estados Unidos \\
\hline 22 & Parent characteristics and early coparenting behavior at the transition to parenthood & 2013 & Estados Unidos \\
\hline 23 & Stability and antecedents of coparenting quality: The role of parent personality and child temperament & 2013 & Estados Unidos \\
\hline 24 & Associations between prenatal coparenting behavior and observed coparenting behavior at 9-months postpartum & 2014 & Estados Unidos \\
\hline 25 & Transitioning to post divorce family life: A grounded theory investigation of resilience in coparenting & 2014 & Estados Unidos \\
\hline 26 & Within-and between-family differences in cooperative and competitive coparenting & 2014 & Estados Unidos \\
\hline 27 & African American couples' coparenting satisfaction and marital characteristics in the first two decades of marriage & 2015 & Estados Unidos \\
\hline 28 & "Either come together or fall apart": Coparenting young children with challenging behaviors & 2015 & Estados Unidos \\
\hline 29 & Marital quality over the transition to parenthood as a predictor of coparenting & 2015 & Estados Unidos \\
\hline
\end{tabular}

* Os estudos descritos nas Tabelas 1, 2 e 3 foram identificados com um código (Id.) na lista de referências e na descrição dos resultados para facilitar sua localização. 
positivamente para o exercício da coparentalidade, (c) variáveis que contribuem negativamente para o exercício da coparentalidade e (d) resultados específicos entre os estudos. As características da relação conjugal antes da chegada do filho reverberam no exercício da coparentalidade e na percepção positiva ou negativa de um parceiro sobre a parentalidade do outro. Para tanto, os estudos encontraram a qualidade do vínculo entre o casal e o investimento na relação, os níveis de satisfação e de conflito conjugal e a capacidade de resolver os problemas no casamento $(3 ; 12 ; 16 ; 18 ; 21$; $22 ; 24 ; 27 ; 28 ; 29)$.

A segunda perspectiva de resultados encontrados nos estudos refere-se à presença de variáveis que contribuem positivamente para o exercício da coparentalidade, prevenindo sabotagem coparental, triangulação, desenvolvimento de comportamentos externalizantes e internalizantes nos filhos, promovendo cooperação e suporte coparental e ajustamento socioemocional nas crianças. As variáveis encontradas nas pesquisas foram qualidade conjugal antes do nascimento do filho, expressividade emocional positiva, brincadeiras e harmonia no ambiente familiar, envolvimento com o filho, incentivo materno à parentalidade do homem, ajustamento conjugal, coabitação entre pais e filhos, temperamento infantil estável, qualidade da coparentalidade com o primeiro filho e foco maior nos filhos em detrimento do casal $(1 ; 3 ; 4 ; 6 ; 8 ; 10 ; 12$; $13 ; 15 ; 19 ; 20 ; 21 ; 22 ; 24 ; 25 ; 26 ; 27)$.

As variáveis que interferem negativamente no exercício da coparentalidade estiveram associadas às seguintes questões: sabotagem, competitividade $\mathrm{e}$ conflito coparentais, baixos níveis de apoio coparental, menor participação masculina na criação dos filhos

TABELA 2

Caracterização do método

\begin{tabular}{|c|c|c|c|}
\hline Id. & Tipo de Pesquisa & Delineamento & Amostra \\
\hline 1 & Quantitativa & Longitudinal e explicativo & 97 casais com filho recém-nascido \\
\hline 2 & Quantitativa & Longitudinal e explicativo & 110 casais à espera do primeiro filho \\
\hline 3 & Quantitativa & Longitudinal, comparativo e explicativo & 177 casais com o primeiro filho adolescente \\
\hline 4 & Quantitativa & Longitudinal e explicativo & 57 casais com dois filhos \\
\hline 5 & Quantitativa & Longitudinal e explicativo & 516 casais com dois filhos adolescentes \\
\hline 6 & Quantitativa & Transversal e explicativo & 104 casais com um filho de até 2 anos \\
\hline 7 & Quantitativa & Transversal e explicativo & 89 casais com o primeiro filho de até 3 anos \\
\hline 8 & Quantitativa & Longitudinal e explicativo & 97 casais com filhos pequenos \\
\hline 9 & Quantitativa & Longitudinal e explicativo & 115 casais à espera do primeiro filho \\
\hline 10 & Quantitativa & Transversal e explicativo & 111 casais com um filho de até 4 anos \\
\hline 11 & Quantitativa & Longitudinal e explicativo & 56 casais com filho recém-nascido \\
\hline 12 & Qualitativa & Transversal e descritivo & 13 pessoas divorciadas - seis pais e sete mães \\
\hline 13 & Quantitativa & Longitudinal e explicativo & 79 casais com filho recém-nascido \\
\hline 14 & Quantitativa & Longitudinal e explicativo & 139 casais com filho recém-nascido \\
\hline 15 & Quantitativa & Longitudinal e explicativo & 88 casais com um filho e a espera do $2^{\circ}$ filho \\
\hline 16 & Quantitativa & Transversal e explicativo & 175 casais com filhos com idade entre 2 e 7 anos \\
\hline 17 & Quantitativa & Longitudinal e explicativo & 150 famílias com o primeiro filho com 1 e 3 meses \\
\hline 18 & Quantitativa & Transversal e explicativo & 519 casais com filhos com idade entre 9 e 13 anos \\
\hline 19 & Quantitativa & Longitudinal e explicativo & 58 casais com filhos pequenos \\
\hline 20 & Quantitativa & Transversal e explicativo & 67 casais com filho com idade entre 16 e 36 meses \\
\hline 21 & Quantitativa & Longitudinal e explicativo & 122 casais com filhos com até 33 meses \\
\hline 22 & Quantitativa & Longitudinal e explicativo & 57 casais à espera do primeiro filho \\
\hline 23 & Quantitativa & Longitudinal e explicativo & 79 casais com filhos pequenos \\
\hline 24 & Quantitativa & Longitudinal e explicativo & 182 casais à espera do primeiro filho \\
\hline 25 & Qualitativa & Transversal e descritivo & 47 pais e mães divorciados exercendo a coparentalidade com o ex-parceiro \\
\hline 26 & Quantitativa & Longitudinal e correlacional & 58 casais com filhos pequenos \\
\hline 27 & Quantitativa & Longitudinal e correlacional & 145 casais com filhos adolescentes \\
\hline 28 & Qualitativa & Transversal e descritivo & 9 casais com filho com idade entre 3 e 6 anos e comportamento opositor \\
\hline 29 & Quantitativa & Longitudinal e explicativo & 96 casais com filhos pequenos \\
\hline
\end{tabular}


e menor suporte a paternidade por parte das mães, reatividade infantil, comportamentos internalizantes nos meninos e comportamentos antissociais em adolescentes. Os resultados apontados foram comportamento irritável ou desadaptativo da criança, baixos níveis de qualidade conjugal, expectativas e crenças negativas ou significativamente diferentes entre o casal durante a gravidez, expressividade emocional negativa, estresse familiar, crítica e desrespeito entre os parceiros no exercício da parentalidade, conflitos conjugais e dificuldades de resolvê-los, dificuldades de sono do bebê, emoções negativas no pré-nascimento, níveis elevados de escolaridade e renda, raiva devido ao divórcio, atitudes incongruentes ou diferentes níveis de disciplina entre pais e mães em relação aos filhos $(1 ; 2 ; 4 ; 5 ; 6 ; 8 ; 9 ; 10 ; 11 ; 13 ; 15 ; 16 ; 17 ; 19 ; 22 ; 23 ; 24$; $25 ; 26 ; 27 ; 28 ; 29)$.
$\mathrm{Na}$ última perspectiva de resultado dos estudos identificaram-se aspectos específicos associados ao exercício da coparentalidade. A escolaridade dos pais foi positivamente associada aos comportamentos de risco dos adolescentes (3), pais demonstraram controle mais negativo do que as mães e sentiram seu papel como menos eficaz $(7 ; 14)$, mães se privam mais de sono devido aos cuidados com os filhos, principalmente durante a noite, e se envolvem mais nos cuidados da prole que os pais $(17 ; 28)$. Além disso, problemas financeiros foram associados positivamente com sintomas depressivos nos participantes (14), pais avaliaram mais positivamente que as mães a qualidade da coparentalidade no período em que os filhos eram bebês (17) e, nos casais divorciados, a qualidade do tempo com o filho foi avaliado como mais relevante que a coabitação (12).

TABELA 3

Objetivos e principais resultados

\begin{tabular}{|c|c|}
\hline Id. & Objetivos \\
\hline 1 & $\begin{array}{l}\text { Verificar como as relações entre irritação e } \\
\text { desadaptabilidade infantil interagem com } \\
\text { a qualidade conjugal e o comportamento } \\
\text { coparental }\end{array}$ \\
\hline 2 & $\begin{array}{l}\text { Verificar a curto e longo prazo as consequências } \\
\text { das expectativas pré-natais dos pais sobre } \\
\text { seu futuro processo familiar e identificar a } \\
\text { estabilidade do apoio coparental }\end{array}$ \\
\hline 3 & $\begin{array}{l}\text { Verificar a associação entre o bem-estar } \\
\text { adolescente, o amor conjugal e a coparen- } \\
\text { talidade e descrever as diferenças entre o pai e a } \\
\text { mãe no que se refere a coparentalidade }\end{array}$ \\
\hline 4 & $\begin{array}{l}\text { Examinar a expressividade emocional parental } \\
\text { como moderadora entre qualidade conjugal e } \\
\text { coparentalidade }\end{array}$ \\
\hline
\end{tabular}

5 Investigar a relação longitudinal do conflito coparental com a parentalidade e o desajuste adolescente ao longo de três anos

6 Verificar se o sexo da criança e a coparentalidade estão associados ao stress familiar e ao ajustamento sócio emocional das crianças

7 Investigar as relações entre parentalidade, coparentalidade e controle de esforço em préescolares

8 Verificar o papel da mãe em relação à participação dos pais e à competência no cuidado da criança

\section{Principais resultados}

(a) Quando a criança apresenta comportamento irritável ou desadaptativo, casais com altos níveis de qualidade conjugal antes do nascimento do filho não apresentam o comportamento de sabotagem coparental; (b) A desadaptabilidade da criança impacta casais com baixa qualidade conjugal, provocando sabotagem e baixos níveis de apoio coparental.

(a) Diferenças significativas nas expectativas dos pais durante a gravidez preveem menor cooperação coparental aos três e doze meses após o nascimento, diminuindo o efeito aos trinta meses; (b) Expectativas e crenças negativas dos pais na gravidez preveem níveis mais baixos de coesão e apoio coparental.

(a) Escolaridade dos pais foi positivamente associada à cooperação coparental e aos comportamentos de risco dos adolescentes; (b) Idade e sexo dos adolescentes foram correlacionados com comportamentos de risco, e sexo, com sintomas depressivos; (c) Comportamentos de risco adolescentes e amor conjugal foram negativamente associados ao conflito coparental.

(a) Expressividade emocional negativa dos pais se associou com conflitos coparentais e a positiva à cooperação coparental; (b) Expressividade negativa do pai foi negativamente associada ao suporte coparental; (c) A expressividade positiva do pai associou-se negativamente à triangulação e a da mãe positivamente com suporte coparental.

(a) Negatividade do pai e da mãe foi preditora de conflito coparental e de comportamento adolescente antissocial; (b) Conflitos coparentais tiveram baixa correlação com desentendimentos conjugais.

(a) Em ambiente familiar em que há brincadeiras, o desajustamento sócio emocional são minimizadas principalmente para meninas; (b) Estresse familiar foi positivamente associado aos comportamentos internalizantes dos meninos; (c) Harmonia familiar foi preditora de ajustamento nas crianças; (d) Cooperação entre os pais e níveis similares de envolvimento com o filho se associaram à menor incidência de problemas internalizantes.

(a) Coparentalidade e parentalidade materna e paterna tiveram fortes associações com controle de esforço; (b) Há diferenças na parentalidade, de pais e mães, em relação ao controle positivo e negativo observado; (c) Pais apresentaram menor controle positivo do que as mães. (d) Coparentalidade apresentou contribuição maior para controle de esforço quando comparada com parentalidade materna e paterna.

(a) O incentivo materno ao exercício parental frente a crenças dos pais sobre o papel paternal e a qualidade global da relação coparental foi associada ao maior envolvimento do pai nos cuidados com a criança; (b) Pais mais críticos com relação à maternagem foram percebidos pelos observadores como menos envolvidos com os filhos do que as mães. 
TABELA 3 (continuação)

\begin{tabular}{cl}
\hline Id. & \multicolumn{1}{c}{ Objetivos } \\
9 & Analisar o afastamento precoce do sistema \\
& coparental e a resolução de problemas sobre \\
& responsabilidades coparentais
\end{tabular}

10 Analisar as relações entre afetividade negativa dos filhos e comportamento de apoio coparental

11 Investigar o papel do temperamento infantil na estabilidade e na mudança na coparentalidade no primeiro ano de vida da criança

12 Compreender o exercício da coparentalidade de pais divorciados

13 Verificar a influência do temperamento infantil na percepção de pais e mães sobre a coparentalidade

14 Verificar se o sexo dos pais, o temperamento da criança e a coparentalidade influenciam questões de estresse, depressão e na eficácia parental na parentalidade precoce

15 Investigar o papel do temperamento da criança para a estabilidade coparental em famílias que estão em transição para o segundo filho

16 Analisar as relações entre conjugalidade, coparentalidade e auto eficácia parental

17 Testar se as dificuldades de sono do bebê predizem a má qualidade de sono e a angústia dos pais e se estão relacionadas à qualidade da coparentalidade

18 Investigar a relação entre a satisfação conjugal e a prática parental dos parceiros considerando o papel da coparentalidade

19 Verificar as associações entre raciocínio indutivo materno, coparentalidade cooperativa e o comportamento pró-social dos filhos

20 Verificar associações diretas entre qualidade coparental, orientações gentis dos pais e competência sócio emocional dos filhos

21 Verificar a percepção coparental de apoio e de confiança como um elo entre a qualidade conjugal e da relação entre pais e filhos

(a) $\mathrm{O}$ afastamento dos pais durante as negociações coparentais se associou à menor afetividade durante o jogo lúdico triádico e com os sentimentos dos pais de que as mães não respeitam sua paternidade; (b) Durante discussões pais que não alcançaram resolução de conflito tenderam a se afastar do parceiro; (c) Afastamento coparental em discussões dificulta a construção de decisões consensuais e foi mais frequente se a criança era do sexo feminino.

(a) Afeto infantil negativo (temperamento difícil) se correlacionou com apoio coparental em famílias com elevados níveis de ajustamento conjugal; (b) Afeto infantil negativo foi preditor significativo de comportamento de sabotagem coparental; (c) Ajustamento conjugal moderou a relação entre afeto infantil negativo e apoio coparental; (d) Afeto infantil negativo se associou negativamente com ajustamento conjugal e foi um fator prejudicial à coparentalidade.

(a) O temperamento foi considerado um moderador da estabilidade longitudinal na coparentalidade; (b) Dificuldade precoce em crianças foi associada com a diminuição dos comportamentos de suporte coparental ao longo do tempo.

(a) Conjugalidade reverbera na coparentalidade considerando o vínculo que uniu o casal, as emoções e a resolução de problemas conjugais; (b) Para as mães, a qualidade do tempo com o filho é mais relevante que a coabitação; (c) Mães tiveram mais práticas educativas individuais e não se consideraram vítimas do divórcio, melhorando a percepção da coparentalidade.

(a) Percepção materna de que o filho tem temperamento positivo foi associado a qualidade coparental; (b) Percepção paterna sobre a coparentalidade foi afetada pelas dificuldades conjugais em período pré-natal; (c) Reatividade infantil foi associada à dificuldade na coparentalidade quando haviam estressores como dificuldades conjugais e parentais.

(a) Foram encontradas interações entre temperamento e coparentalidade em homens e mulheres que se adaptaram ao seu papel parental; (b) Problemas financeiros se associaram positivamente com sintomas depressivos, mas não com a eficácia parental ou stress; (c) O sentimento de eficácia parental é menor em pais se comparados às mães.

(a) A cooperação aumenta ao longo do tempo, sendo que as mães pontuaram mais para qualidade coparental após o nascimento do segundo filho; (b) Em observações familiares as tríades tiveram menor nível de cooperação se comparadas aos quartetos; (c) O temperamento estável do segundo filho está associado a boa relação coparental; (d) A coparentalidade com o primeiro filho é preditora de como será com o segundo filho.

(a) Investimento na conjugalidade foi associado positivamente com a auto eficácia parental; (b) Satisfação conjugal relatada pelas mães foi negativamente associada à auto eficácia parental dos pais; (c) Dificuldades na coparentalidade foram associadas à diminuição da auto eficácia parental e maior satisfação conjugal de acordo com os pais.

(a) Mães se privam mais do sono devido aos cuidados com os filhos, principalmente durante a noite; (b) Pais avaliaram melhor do que as mães a qualidade da coparentalidade no período em que os filhos eram bebês; (c) Dificuldades de sono do bebê geram má qualidade do sono, sintomas depressivos e avaliação negativa da coparentalidade; (d) Sintomas depressivos foram mais fortes para as mães do que para os pais entre o primeiro e o terceiro mês de vida da criança.

(a) A parentalidade materna provocou efeitos indiretos na satisfação conjugal dos pais e no apoio emocional das mães; (b) $\mathrm{O}$ conflito dos pais se associou com a satisfação conjugal dos mesmos e à rejeição materna; (c) A satisfação conjugal e a rejeição das mães, o apoio emocional dos pais se associaram à contribuição das mães para o conflito; (d) A satisfação conjugal foi associada às contribuições para a coparentalidade e para as práticas parentais dos casais.

(a) Coparentalidade cooperativa foi positivamente associada com o comportamento pró-social das crianças; (b) Baixos níveis de comportamento pró-social dos filhos ocorreram quando havia níveis baixos de cooperação coparental e em mães que utilizavam o raciocínio indutivo com menor frequência.

(a) Mediação entre qualidade coparental e competências socioemocionais nos filhos através da orientação gentil parental para mães; (b) Mães que receberam apoio coparental agiram de forma mais gentil ao dar orientações aos, filhos, que desenvolveram níveis maiores de competência socioemocional.

(a) Qualidade conjugal foi relacionada à maior qualidade da relação mãe-filho via percepções coparentais maternas mais positivas; (b) Qualidade conjugal foi relacionada à maior qualidade da relação pai-filho e pai-filha via percepção coparental paterna mais positiva; (c) Qualidade conjugal e qualidade da relação mãe-filho se associaram através da percepção positiva da coparentalidade de mães e pais.

(continua) 
TABELA 3 (conclusão)

\begin{tabular}{ll}
\hline Id. & \multicolumn{1}{c}{ Objetivos } \\
\hline 22 & $\begin{array}{l}\text { Investigar a relação coparental e examinar } \\
\text { emoções negativas dos pais, percepções de } \\
\text { aceitação materna na infância e crenças das } \\
\text { mães sobre o papel coparental dos pais }\end{array}$
\end{tabular}

23 Verificar se a personalidade dos pais e o temperamento infantil se associam com o desenvolvimento e a estabilidade coparental durante os três primeiros anos da criança

24 Testar se o comportamento coparental (medido com um programa de estimulação à coparentalidade) é preditor de comportamento coparental pós-natal

25 Compreender os processos de resiliência e a coparentalidade pós-divórcio

26 Investigar variações da coparentalidade entre famílias de diversos contextos

27 Mapear a trajetória de satisfação coparental durante a primeira e a segunda décadas de casamento considerando as variáveis amor e conflito conjugal

28 Verificar a coparentalidade de casais com crianças pequenas que apresentam comportamento opositor

29 Investigar se mudanças na qualidade conjugal de pais se relacionam com a qualidade coparental até os dois anos de idade dos filhos

(a) Casais com mais dificuldades coparentais no pós-parto foram aqueles com níveis maiores de emoções negativas no pré-nascimento; (b) A crença das mães sobre a importância do papel dos pais foi preditora significativa de apoio coparental e moderou a conjugalidade pré-nascimento; (c) Pais e mães com níveis elevados de escolaridade e renda tiveram mais comportamentos negativos após o nascimento da criança.

(a) As dimensões de personalidade dos pais, comunhão e emoções negativas, foram diretamente associadas à qualidade coparental; (b) Emoções negativas das mães foram associadas à maior qualidade coparental; (c) O temperamento da criança e a personalidade dos pais interagem na previsão da relação coparental nos primeiros anos de vida da criança.

(a) A relação coparental se constitui antes do nascimento da criança; (b) Casais que apresentaram qualidade no comportamento coparental pré-natal tiveram níveis elevados em cooperação, calor familiar e comportamento lúdico; (c) O modelo que inclúa o comportamento coparental pré-natal como preditor de comportamento coparental após nove meses, mostrou-se bem ajustado, explicando $19 \%$ da variância do comportamento solidário coparental e 14\% da variância do comportamento de sabotagem.

(a) Pais focados nas crianças regularam suas respostas emocionais e debateram sobre tempo e dinheiro foram mais eficazes que aqueles que sentiam raiva em função do divórcio ou se envolviam em conflitos frequentes com o ex-cônjuge; (b) Pais que se preocupam com a regulação emocional dos filhos procuram não falar negativamente sobre os ex-parceiros na frente de seus filhos.

(a) Casais com maiores níveis de conflito conjugal e de comportamentos centrados na criança foram menos cooperativas; (b) $\mathrm{O}$ sexo da criança e o amor conjugal não foram associados com a cooperação coparental; (c) Famílias centradas nas crianças tiveram níveis mais baixos de competição coparental.

(a) Amor conjugal (relação positiva) e conflito conjugal (relação negativa) estão associados à satisfação coparental ao longo do tempo; (b) Casais com mais atitudes incongruentes em relação aos filhos apresentaram menores níveis de amor conjugal e de satisfação coparental; (c) Pais e mães são mais suscetíveis aos problemas no casamento, o que ocasiona uma diminuição da satisfação coparental.

(a) Pais de filhos com comportamento opositor relataram se sentir perdidos e desamparados em relação às dificuldades dos filhos; (b) Nos casais em que existia sabotagem coparental os pais eram mais brandos com os limites do que as mães; (c) Casais com maiores níveis de conflito expressaram ansiedade sobre o impacto a longo prazo dos conflitos coparentais no filho; (d) Na divisão de trabalho coparental os pais precisavam ser convidados pelas mães.

(a) Declínio na satisfação conjugal prediz maior competitividade coparental, menor apoio coparental do pais e participação na criação dos filhos; (b) Conflito conjugal é preditor de níveis baixos de apoio coparental e menor suporte à paternidade dos pais pelas mães; (c) Qualidade conjugal tem efeitos diretos sobre a qualidade coparental para os pais.

\section{Discussão e Considerações finais}

O objetivo deste artigo foi mapear a produção científica de estudos empíricos sobre coparentalidade e analisar suas características, objetivos e principais resultados. Com relação à quantidade de estudos e ao ano de publicação, em um período de dez anos (2007 a agosto de 2016) foram publicados 29 artigos. Embora a distribuição dos estudos não seja equitativa, a média de publicação é de aproximadamente três artigos por ano. Considerando o período de busca, o número total e a média de estudos publicados por ano é relativamente baixo. Além disso, 25 estudos, que correspondem à $86 \%$, foram realizados nos Estados Unidos e apenas um no Brasil, refletindo a urgência de mais investimento em pesquisas sobre a referida temática no contexto brasileiro (Frizzo et al., 2005; Lamela, Nunes-Costa, \& Figueiredo, 2010).

Os pesquisadores avaliaram a coparentalidade predominantemente por meio de pesquisa quantitativa, longitudinal e explicativa, característica que pode indicar avanços em termos de conhecimento científico sobre a temática que já não se encontra mais em fase exploratória. Ainda, esse dado pode refletir a complexidade do próprio fenômeno, em que uma quantidade expressiva de variáveis se inter-relacionam recursivamente e, portanto, necessitam de métodos que testem correlação, predição e causalidade entre as mesmas por meio de estudos explicativos e longitudinais com famílias. Sendo que a investigação 
da temática por meio de delineamentos complexos pode também estar associada ao número relativamente baixo de estudos encontrados.

O tipo de amostra que compôs quase que a totalidade dos estudos variou da seguinte forma: casais grávidos (quatro), casais com filhos recém-nascidos (cinco), casais com filhos pequenos (doze) e casais com filhos adolescentes (quatro). Esse resultado indica que os pesquisadores têm investigado os diferentes estágios de exercício da coparentalidade, sendo possível identificar alterações associadas ao desenvolvimento da criança ou à relação entre o casal, como pais e cônjuges.

Por meio da análise do objetivo principal dos estudos, percebe-se que a literatura científica tem centrado esforços para verificar possíveis associações e impacto das características do comportamento infantil no exercício da coparentalidade e na relação conjugal, bem como, repercussões como estresse familiar, depressão e angústia nos pais, conforme apontado por Majdandzic et al. (2012). Outra onda de estudos tem focalizado nas possíveis inter-relações entre características da conjugalidade, tais como, níveis de conflito e satisfação conjugal e capacidade de resolução de problemas, com responsabilidade, apoio e confiança coparental, eficácia parental e sentimentos em relação aos filhos. A relação do casal antes e depois da chegada do primeiro e do segundo filho, as expectativas e crenças dos pais sobre as relações familiares e o exercício da coparentalidade, as diferenças entre pais e mães e a coparentalidade no contexto do divórcio também tem sido foco dos pesquisadores.

A multiplicidade de perspectivas de investigação do fenômeno pode estar associada aos questionamentos que ainda buscam ser respondidos pelos estudiosos da temática. Podem refletir também as diferentes formas de compreender os processos familiares, especialmente, as transições que ocorrem nos diferentes estágios do ciclo vital em que os parceiros, inicialmente, exercendo o papel de cônjuges, tornam-se pais e co-cuidadores, e os possíveis impactos que o surgimento de dois novos subsistemas, o parental e o coparental, com o acréscimo das respectivas funções, provocam aos indivíduos e à relação do casal.

Conforme as pesquisas (Frizzo et al., 2005; Grzybowski \& Wagner 2010; Lamela, Figueiredo, \& Bastos, 2010; Lamela, Figueiredo, \& Bastos, 2013; Lamela, Nunes-Costa, \& Figueiredo, 2010), os resultados dos estudos têm demonstrado que dificuldades conjugais tendem a se refletir em problemas no exercício da coparentalidade. Esse resultado, acerca do qual há relativo consenso na literatura, pode não ser observado com clareza pelos casais em geral. Nesse sentido, ainda que comprovado pelos estudos, é fundamental tratar a temática preventivamente. Tal se faz necessário porque a chegada de um filho tende a desorganizar o casal inicialmente, exigindo maior esforço dos parceiros para se manterem próximos como marido e esposa. Dessa forma, se o vínculo estiver frágil e a relação for excessivamente conflituosa, tenderá a se agravar diante dos desafios da parentalidade e da coparentalidade.

Os resultados dos estudos que compõem esta revisão sistemática fornecem evidências de que qualidade e ajustamento conjugal antes do nascimento do filho, expressão de sentimentos positivos, brincadeiras e harmonia no ambiente familiar, envolvimento, atenção e coabitação entre pais e filhos, valorização à parentalidade do parceiro e estabilidade do temperamento da criança são preditores de qualidade coparental. Esses resultados apontam uma série de fatores relacionados ao ambiente familiar e ao temperamento da criança que, embora possam ter sido herdados dos genitores, interferem no exercício da coparentalidade. Tal poderia explicar, por exemplo, porque casais que referem qualidade conjugal, equilíbrio nos cuidados com a prole, condições materiais e emocionais para exercer o papel de pais, enfrentam significativas dificuldades com os filhos, mesmo em condições adequadas para o desenvolvimento destes.

Nessa perspectiva, são apontados os problemas enfrentados pelos pais no exercício da coparentalidade como decorrentes do comportamento irritável ou desadaptativo da criança. Outros fatores referem-se aos problemas na área conjugal, às expectativas anteriores ao nascimento da criança, aos problemas entre os parceiros no exercício da parentalidade e à escolaridade e renda dos pais (Lamela, Nunes-Costa, \& Figueiredo, 2010; Margolin et al., 2001). Alguns estudos apontam também diferenças entre pais e mães tanto na prestação de cuidados com os filhos, quanto na percepção e no impacto sofrido pelas dificuldades oriundas da parentalidade e da coparentalidade.

Os resultados dos estudos revisados apontam diferentes fatores associados tanto às dificuldades no exercício da coparentalidade quanto à qualidade neste e nos demais subsistemas familiares. É consenso que problemas internalizantes e externalizantes na prole ocorrem mais em ambientes perpassados por invalidação, desarmonia, conflitos excessivos e sem resolução, negatividade e características emocionais e comportamentais de pais e filhos (Mosmann et al., no prelo; Teubert \& Pinquart, 2010).

De acordo com essa revisão sistemática, percebe-se que atuar em nível de prevenção junto aos casais que pretendem ser pais, no desenvolvimento de habilidades no exercício da parentalidade e na importância da 
gestão congruente e validante da coparentalidade são essenciais ao desenvolvimento adequado dos filhos (Frizzo et al., 2005). Outro aspecto importante refere-se ao fato de os estudos empíricos analisados investigarem apenas a coparentalidade de casais heterossexuais. Não foram encontrados estudos avaliando esse subsistema em casais de pessoas do mesmo sexo ou por duplas de cuidadores sem vínculo conjugal. Realizar pesquisas com esses grupos, pode apontar diferentes características do exercício da coparentalidade.

Essa revisão reúne resultados de estudos potenciais da área de família e pode ser útil especialmente para os profissionais da área clínica atendendo famílias que enfrentam as dificuldades apontadas ao longo deste estudo, bem como, pesquisadores brasileiros que desejam conhecer o estado da arte da referida temática e empreender novas investigações. Entre as limitações deste estudo cita-se a impossibilidade de discutir separadamente muitos dos resultados encontrados, sendo necessário reuni-los em uma mesma perspectiva e analisá-los de forma integrada. Além disso, resultados como às associações positivas entre escolaridade e renda e os problemas de comportamento adolescente não foram discutidos por não estarem diretamente relacionados à coparentalidade (Feinberg, 2003). Dessa forma, sugere-se que outros estudos possam discutir tais questões de forma crítica e, no Brasil, que os pesquisadores se dediquem à investigação da coparentalidade e dos fatores que reverberam de forma tanto positiva quanto negativa em todo o ambiente familiar.

\section{Referências}

Altenburger, L. E., Schoppe-Sullivan, S. J., Lang, S. N., Bower, D. J., \& Dush, C. M. K. (2014). Associations between prenatal coparenting behavior and observed coparenting behavior at 9-months postpartum. Journal of Family Psychology, 28(4), 495-504. https://doi.org/10.1037/fam0000012

Baril, M. E., Crouter, A. C., \& McHale, S. M. (2007). Processes linking adolescent well-being, marital love, and coparenting. Journal of Family Psychology, 21(4), 645-654. https://doi.org/10.1037/0893-3200.21.4.645

Blandon, A. Y., Scrimgeour, M. B., Stifter, C. A., \& Buss, K. A. (2014). Within-and between-family differences in cooperative and competitive coparenting. Journal of Family Psychology, 28(1), 106-111. https://doi.org/10.1037/ a0035266

Burney, R. V. \& Leerkes, E. M. (2010). Links between mothers' and fathers' perceptions of infant temperament and coparenting. Infant Behavior and Development, 33(2), 125-135. https://doi.org/10.1016/j.infbeh.2009.12.002

Christopher, C., Umemura, T., Mann, T., Jacobvitz, D., \& Hazen, N. (2015). Marital Quality over the Transition to Parenthood as a Predictor of Coparenting. Journal of Child and Family Studies, 24(12), 3636-3651. https://doi. org/10.1007/s10826-015-0172-0

Cook, J. C., Schoppe-Sullivan, S. J., Buckley, C. K., \& Davis, E. F. (2009). Are some children harder to coparent than others? Children's negative emotionality and coparenting relationship quality. Journal of Family Psychology, 23(4), 606-610. https://doi.org/10.1037/a0015992

Costa, A. B., Zoltowski, A. P. C., Koller, S. H., \& Teixeira, M. A. P. (2015). Construção de uma escala para avaliar a qualidade metodológica de revisões sistemáticas. Ciências \& Saúde Coletiva, 20(8), 2441-2452. https://doi. org/10.1590/1413-81232015208.10762014

Davis, E. F., Schoppe-Sullivan, S. J., Mangelsdorf, S. C., \& Brown, G. L. (2009). The role of infant temperament in stability and change in coparenting across the first year of life. Parenting: Science and Practice, 9(1-2), 143-159. https://doi.org/10.1080/15295190802656836

Elliston, D., McHale, J., Talbot, J., Parmley, M., \& Kuersten-Hogan, R. (2008). Withdrawal from coparenting interactions during early infancy. Family Process, 47(4), 481-499. https://doi.org/10.1111/j.1545-5300.2008.00267.x

Feinberg, M. E. (2003). The Internal structure and ecological context of coparenting: a framework for research and intervention. Parenting: Science and Practice, 3(2), 95-131. https://doi.org/10.1111/j.1741-3737.2007.00400.x

Feinberg, M. E., Kan, M. L., \& Hetherington, E. M. (2007). The longitudinal influence of coparenting conflict on parental negativity and adolescent maladjustment. Journal of Marriage and Family, 69(3), 687-702. doi: 10.1111/j.17413737.2007.00400.x

Fernández-Ríos, L. \& Buela-Casal, G. (2009). Standards for the preparation and writing of psychology review articles. International Journal of Clinical and Health Psychology, 9, 329-344. Retrieved from http://hdl.handle.net/10481/33048

Frizzo, G. B., Kreutz, C. M., Schmidt, C., Piccinini, C. A., \& Bosa, C. (2005). O Conceito de coparentalidade e suas implicações para a pesquisa e para a clínica. Revista Brasileira de Crescimento e Desenvolvimento Humano, 15(3), 84-93. Retrieved from http://pepsic.bvsalud.org/pdf/rbcdh/v15n3/10.pdf

Grzybowski, L. S. \& Wagner, A. (2010). Casa do pai, casa da mãe: A coparentalidade após o divórcio. Psicologia: Teoria e Pesquisa, 26(1), 77-87. https://doi.org/10.1590/S0102-37722010000100010

Holland, A. S. \& McElwain, N. L. (2013). Maternal and paternal perceptions of coparenting as a link between marital quality and the parent - toddler relationship. Journal of Family Psychology, 27(1), 117-126. https://doi.org/10.1037/ a0031427 
Jamison, T. B., Coleman, M., Ganong, L. H., \& Feistman, R. E. (2014). Transitioning to post divorce family life: A grounded theory investigation of resilience in coparenting. Family Relations, 63(3), 411-423. https://doi.org/10.1111/ fare. 12074

Karreman, A., Van Tuijl, C., Van Aken, M. A. G., \& Deković, M. (2008). Parenting, coparenting, and effortful control in preschoolers. Journal of Family Psychology, 22(1), 30-40. https://doi.org/10.1037/0893-3200.22.1.30

Kolak, A. M. \& Vernon-Feagans, L. (2008). Family-level coparenting processes and child gender as moderators of family stress and toddler adjustment. Infant and Child Development, 17(6), 617-638. https://doi.org/10.1002/icd.577

Kolak, A. M. \& Volling, B. L. (2007). Parental expressiveness as a moderator of coparenting and marital relationship quality. Family Relations, 56(5), 467-478. https://doi.org/10.1111/j.1741-3729.2007.00474.x

Kwon, K. A., Jeon, H. J., \& Elicker, J. (2013). Links among coparenting quality, parental gentle guidance, and toddlers' social emotional competencies: Testing direct, mediational, and moderational models. Journal of Family Studies, 19(1), 19-34. https://doi.org/10.5172/jfs.2013.19.1.19

Lamela, D., Figueiredo, B., \& Bastos, A. (2010). Adaptação ao divórcio e relações coparentais: contributos da teoria da vinculação. Psicologia: Reflexão e Crítica, 23(3), 562-574. https://doi.org/10.1590/S0102-79722010000300017

Lamela, D., Nunes-Costa, R., \& Figueiredo, B. (2010). Modelos teóricos das relações coparentais: revisão crítica. Psicologia em Estudo, 15(1), 205-216. https://doi.org/10.1590/S0102-79722013000100003

Lamela, D., Figueiredo, B., \& Bastos, A. (2013). Perfis de vinculação, coparentalidade e ajustamento familiar em pais recém-divorciados: diferenças no ajustamento psicológico. Psicologia: Reflexão e Crítica, 26(1), 19-28. http://dx.doi org/10.1590/S0102-79722013000100003

Laxman, D. J., Jessee, A., Mangelsdorf, S. C., Rossmiller-Giesing, W., Brown, G. L., \& Schoppe-Sullivan, S. J. (2013). Stability and antecedents of coparenting quality: The role of parent personality and child temperament. Infant Behavior and Development, 36(2), 210-222. https://doi.org/10.1016/j.infbeh.2013.01.001

Majdandzic, M., Vente, W., Feinberg, M. E., Aktar, E., \& Bögels S. M. (2012). Bidirectional associations between coparenting relations and family member anxiety: a review and conceptual model. Clinical Child and Family Psychology Review, 15(1): 28-42. https://doi.org/10.1007/s10567-011-0103-6

Margolin, G., Gordis, E. B., \& John, R. S. (2001). Coparenting: a link between marital conflict and parenting in two parent families. Journal of family Psychology, 15(1), 3-21. doi: https://doi.org/10.1037/0893-3200.15.1.3

McDaniel, B. T. \& Teti, D. M. (2012). Coparenting quality during the first three months after birth: The role of infant sleep quality. Journal of Family Psychology, 26(6), 886-895. https://doi.org/10.1037/a0030707

McHale, J. P., Kuersten-Hogan, R. \& Rao, N. (2004). Growing points for coparenting theory and research. Journal of Adult Development, 11(3), 221-234. https://doi.org/10.1023/B:JADE.0000035629.29960.ed

McHale, J. P. \& Rotman, T. (2007). Is seeing believing? Expectant parents' outlooks on coparenting and later coparenting solidarity. Infant Behavior and Development, 30(1), 63-81. https://doi.org/10.1016/j.infbeh.2006.11.007

Mendez, L. M. R., Loker, T., Fefer, S., Wolgemuth, J., \& Mann, A. (2015). "Either come together or fall apart": Coparenting young children with challenging behaviors. Couple and Family Psychology: Research and Practice, 4(2), 74-91. https://doi.org/10.1037/cfp0000039

Merrifield, K. A. \& Gamble, W. C. (2012). Associations among marital qualities, supportive and undermining coparenting, and parenting self-efficacy: Testing spillover and stress-buffering processes. Journal of Family Issues, 34(4), $510-533$. https://doi.org/10.1177/0192513X12445561

Mosmann, C. P., Costa, C. B., Einsfeld, P., Silva, A. G. M., \& Koch, C. (no prelo). Conjugalidade, parentalidade e coparentalidade: associações com sintomas externalizantes e internalizantes em crianças e adolescentes. Estudos de Psicologia (PUCCampinas).

Pedro, M. F., Ribeiro, T., \& Shelton, K. H. (2012). Marital satisfaction and partners' parenting practices: The mediating role of coparenting behavior.Journal of Family Psychology, 26(4), 509-522. https://doi.org/10.1037/a0029121

Riina, E. M. \& McHale, S. M. (2015). African american couples' coparenting satisfaction and marital characteristics in the first two decades of marriage. Journal of Family Issues, 36(7) 902-923. https://doi.org/10.1177/0192513X 13495855

Schoppe-Sullivan, S. J., Brown, G. L., Cannon, E. A., Mangelsdorf, S. C., \& Sokolowski, M. S. (2008). Maternal gatekeeping, coparenting quality, and fathering behavior in families with infants. Journal of Family Psychology, 22(3), 389-398. https://doi.org/10.1037/0893-3200.22.3.389

Schoppe-Sullivan, S. J., Mangelsdorf, S. C., Brown, G. L., \& Sokolowski, M. S. (2007). Goodness-of-fit in family context: Infant temperament, marital quality, and early coparenting behavior. Infant Behavior and Development, 30(1), 82-96. https://doi.org/10.1016/j.infbeh.2006.11.008

Schoppe-Sullivan, S. J. \& Mangelsdorf, S. C. (2013). Parent characteristics and early coparenting behavior at the transition to parenthood. Social Development, 22(2), 363-383. https://doi.org/10.1111/sode.12014

Scrimgeour, M. B., Blandon, A. Y., Stifter, C. A., \& Buss, K. A. (2013). Cooperative coparenting moderates the association between parenting practices and children's prosocial behavior. Journal of Family Psychology, 27(3), 506-511. https://doi.org/10.1037/a0032893

Sifuentes, M. \& Bosa, C. A. (2010). Criando pré-escolares com autismo: características e desafios da coparentalidade. Psicologia em Estudo, 15(3), 477-48. http://dx.doi.org/10.1590/S1413-73722010000300005 
Solmeyer, A. R. \& Feinberg, M. E. (2011). Mother and father adjustment during early parenthood: The roles of infant temperament and coparenting relationship quality. Infant Behavior and Development, 34(4), 504-514. https://doi. org/10.1016/j.infbeh.2011.07.006

Szabó, N., Dubas, J. S., \& Van Aken, M. A. G. (2012). And baby makes four: The stability of coparenting and the effects of child temperament after the arrival of a second child. Journal of Family Psychology, 26(4), 554-564. https://doi. org/10.1037/a0028805

Teubert, D. \& Pinquart, M. (2010). The association between coparenting and child adjustment: A meta-analysis. Parenting, 10(4), 286-307. https://doi.org/10.1080/15295192.2010.492040

Van Egeren, L. A. \& Hawkinjs, D. P. (2004). Coming to terms with coparenting: implications of definition and measurement. Journal of Adult Development, 11(3), 165-178. https://doi.org/10.1023/B:JADE.0000035625.74672.0b

Autores:

Crístofer Batista da Costa - Mestre, Universidade do Vale do Rio dos Sinos.

Mariana Rodrigues Machado - Especialista, Universidade do Vale do Rio dos Sinos.

Mariana Cunha Schneider - Graduando, Universidade do Vale do Rio dos Sinos.

Clarisse Pereira Mosmann - Doutora, Universidade do Vale do Rio dos Sinos.

Endereço para correspondência

Crístofer Batista da Costa

Rua Marcelo Gama 1440/304

90540041 - Porto Alegre, RS, Brasil

<cristoferbatistadacosta@gmail.com>

Recebido em: 29.09 .2016

Aceito em: 04.04.2017 